\title{
An efficient synthesis of julibrosides related to neosaponin
}

\author{
Yuguo Du, Meimei Zhang and Fanzuo Kong* \\ Research Center for Eco-Environmental Sciences, Chinese Academy of Sciences, P.O. Box 2871, \\ Beijing 100085, China
}

Received (in Cambridge, UK) 12th December 2000, Accepted 24th July 2001

First published as an Advance Article on the web 23rd August 2001

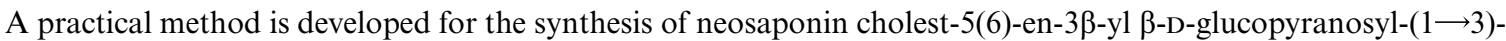
$[\alpha$-L-arabinofuranosyl- $(1 \longrightarrow 4)]-\alpha$-L-rhamnopyranosyl- $(1 \longrightarrow 2)-\beta-D-$ glucopyranoside. This julibroside mimic shows cytotoxic activity towards P388 (mouse leukaemia) with $\mathrm{GI}_{50}=22.5 \mu \mathrm{g} \mathrm{mL}{ }^{-1}$.
\end{abstract}

\begin{abstract}
Introduction
Albizia julibrissin is a plant widely distributed in China. The stem bark of the plant has been used as a sedative and antiinflammatory drug to treat lung swellings and pains, skin ulcers, and wounds. ${ }^{1}$ Julibrosides, isolated triterpenoid glycosides from Albizia julibrissin, show cytotoxicity against KB cells. ${ }^{2}$ When their ester-linked tetrasaccharide was removed, the cytotoxicity dramatically decreased. ${ }^{3}$ This finding prompted us to prepare neosaponins carrying the original tetrasaccharide component and to examine their biological activities.

Although the tetrasaccharide has been prepared by lithium iodide-promoted ester cleavage of julibrosides, its final glycosylation with an aglycone is still troublesome. ${ }^{2 b, 4}$ Many reports revealed that coupling of the aglycone with a glycosyl donor without the participation of a neighboring group produced a mixture of $\alpha$ and $\beta$ anomers, which were difficult to separate. ${ }^{4,5}$ Our method is to connect the endo-glucose unit to the aglycone moiety at the very beginning in order to get exclusive $\beta$-linkage, then manipulate the glucose on $\mathrm{C}-2$ and extend the sugar chain subsequently. ${ }^{6}$
\end{abstract}

\section{Results and discussion}

In our previous synthesis of $1 \longrightarrow 3$-linked rhamnopyranoside, ${ }^{7 a}$ we showed that chemoselective deacetylation in the presence of the benzoyl group could be best controlled under acidic conditions. ${ }^{8}$ Thus, orthoester $\mathbf{1} \mathbf{b}^{9}$ was treated with $95 \%$ aq. acetic acid, ${ }^{10}$ followed by Schmidt activation ${ }^{11}$ on the anomeric center using trichloroacetonitrile and 1,8-diazabicyclo[5.4.0]undec-7ene (DBU), to give 2- $O$-acetyl-3,4,6-tri- $O$-benzoyl- $\alpha$-D-glucopyranosyl trichloroacetimidate 2 ( $87 \%$ yield for two steps) (Scheme 1). Coupling of $\mathbf{2}$ with cholesterol $\mathbf{3}$ in anhydrous $\mathrm{CH}_{2} \mathrm{Cl}_{2}$ in the presence of TMSOTf (0.1 equiv.) furnished exclusively $\beta$-D-glucopyranoside 4 in $91 \%$ yield. The doublet at $\delta 4.79$ with coupling constant of $7.9 \mathrm{~Hz}$ clearly showed the $\beta$ linkage between the glucopyranosyl moiety and the cholesterol residue. It is noteworthy that attempts using the corresponding bromide as the donor generated orthoester as a significant byproduct while the Helfrich reaction ${ }^{12}$ of 1,2 -di- $O$-acetyl-3,4,6tri- $O$-benzoyl- $\beta$-D-glucopyranose with 3 gave only a $40 \%$ yield of desired compound $\mathbf{4}$. Regioselective deacetylation of $\mathbf{4}$ with $3 \%$ acetyl chloride in $\mathrm{MeOH}-\mathrm{CH}_{2} \mathrm{Cl}_{2}(1: 1)$ co-solvent was smoothly carried out and 2-OH acceptor $\mathbf{5}$ was provided in $89 \%$ yield as a white solid.

Initial attempts at the synthesis of trisaccharide donor $\mathbf{1 8}$ followed our previous strategy of sugar-sugar orthoester formation-rearrangement. However, TMSOTf-catalyzed re- arrangement of $\mathbf{6}$ was surprisingly unpractical for gram-scale preparation of $\mathbf{8 b}$ since an unidentified by-product was obtained in significant quantities. Thus, the 4-OH of the rhamnopyranosyl residue in the disaccharide orthoester $6^{7 a}$ was protected with the chloroacetyl group using chloroacetic anhydride in pyridine to give 7 , treatment of which with TMSOTf gave 8a in 77\% yield. Unfortunately, cleavage of the chloroacetyl group of 8a was quite difficult and a low yield of $\mathbf{8 b}$ was given although several reaction conditions were investigated, including the use of thiourea- $\mathrm{NaHCO}_{3}-\mathrm{EtOH}$, thiourea-lutidine-EtOH, thiourea-Et ${ }_{3} \mathrm{~N}-1,4$-dioxane, and ethylenediamine-Pyr in this reaction. ${ }^{13}$

Alternatively, the $\alpha$-L-arabinofuranosyl trichloroacetimidate $\mathbf{9}^{7 b}$ and $\alpha$-L-rhamnopyranoside acceptor $\mathbf{1 0}^{14}$ were coupled first under standard glycosylation conditions ( 0.05 equiv. of TMSOTf, $\mathrm{CH}_{2} \mathrm{Cl}_{2}, 0{ }^{\circ} \mathrm{C}, 1$ hour) to give disaccharide 11 in $83 \%$ yield. Hydrolysis of $\mathbf{1 1}$ with $90 \%$ trifluoroacetic acid (TFA) gave diol 12 in $88 \%$ yield. At this stage, 2,3-selective glycosylation on disaccharide 12 gave mainly $1 \longrightarrow 2$-linked product. ${ }^{7 a}$ To overcome this problem, regioselective benzoylation of $\mathbf{1 2}$ was tried with 1.05 equiv. of $\mathrm{BzCl}$ in pyridine in an ice-water-bath, and 2-O-benzoylated product 13 was obtained in $79 \%$ yield as an amorphous solid. The chemical shifts of $\mathrm{H}-2\left(\delta 5.36, J_{1,2}\right.$ $\left.1.4, J_{2,3} 3.3 \mathrm{~Hz}\right)$ and $\mathrm{H}-3\left(\delta 4.39, J_{2,3} 3.3, J_{3,4} 9.3 \mathrm{~Hz}\right)$, based on 2D ${ }^{1} \mathrm{H}^{-1} \mathrm{H}$ COSY analysis, assured the regioselectivity at $\mathrm{C}-2$ of 12. Coupling of fully acetylated glucopyranosyl trichloroacetimidate $\mathbf{1 4}$ with disaccharide acceptor $\mathbf{1 3}$ in anhydrous $\mathrm{CH}_{2} \mathrm{Cl}_{2}$ using a catalytic amount of TMSOTf ( 0.1 equiv.) at $0{ }^{\circ} \mathrm{C}$ for 2 hours gave trisaccharide $\mathbf{1 5}$ as the major product $(63 \%)$, together with $20 \%$ of orthoester 16 . The orthoester formation was found to be dependent on the amount of promoter used in the reaction and could be well depressed by adding another portion of TMSOTf ( 0.03 equiv.). Thus, trisaccharide $\mathbf{1 5}$ was prepared in $78 \%$ yield without formation of detectable orthoester within 4 hours. Trisaccharide imidate 18 was prepared from 15 in two steps (deallylation to 17: $\mathrm{PdCl}_{2}, \mathrm{NaOAc}$, HOAc; activation: $\mathrm{Cl}_{3} \mathrm{CCN}, \mathrm{DBU}, \mathrm{CH}_{2} \mathrm{Cl}_{2}$ ) in a total yield of $55 \%$. TMSOTf-catalyzed glycosylation of $\mathbf{1 8}$ and $\mathbf{5}$ in $\mathrm{CH}_{2} \mathrm{Cl}_{2}$ finished assembly of this neosaponin derivative $19(86 \%)$ whose structure was determined with the help of ${ }^{1} \mathrm{H}$ NMR, ${ }^{13} \mathrm{C}$ NMR and ${ }^{1} \mathrm{H}-{ }^{1} \mathrm{H},{ }^{1} \mathrm{H}-{ }^{13} \mathrm{C}$ COSY spectra. Treatment of fully acylprotected 19 with $\mathrm{NaOMe}$ in $\mathrm{MeOH}$ afforded the designed neosaponin $\mathbf{2 0}$ in $95 \%$ yield.

The cytotoxicity of the neosaponin $\mathbf{2 0}$ was preliminarily examined by incubation of the P388 cell line with 20 at $37^{\circ} \mathrm{C}$ for 72 hours in a $5 \% \mathrm{CO}_{2}$ incubator. ${ }^{15}$ The plate was read on a microplate reader at a wavelength of $540 \mathrm{~nm}$. Neosaponin 20 showed cytotoxic activity on inhibition of P388 cell growth 
increase at $\mathrm{GI}_{50} 22.5 \mu \mathrm{g} \mathrm{mL} \mathrm{mL}^{-1}$. This result demonstrated Nohara's idea that the tetrasaccharide moiety of julibrosides plays an important role in the expression their cytotoxic activities. $^{2 b}$ Synthesis of the tetrasaccharide-containing neosaponin analogues and the testing of their bioactivities are currently underway in our group.

In summary, we present here a convergent synthesis of a julibroside-related neosaponin showing cytotoxic activity towards P388. This concise and practical synthetic strategy should provide the possibility for the synthesis of other analogues.

\section{Experimental}

Optical rotations were determined at $25^{\circ} \mathrm{C}$ with a Perkin-Elmer Model 241-Mc automatic polarimeter, and $[\alpha]_{\mathrm{D}}$-values are in units of $10^{-1} \mathrm{deg} \mathrm{cm}^{2} \mathrm{~g}^{-1}$. Mps were determined with a 'Mel-Temp' apparatus. ${ }^{1} \mathrm{H}$ NMR,,${ }^{13} \mathrm{C}$ NMR and ${ }^{1} \mathrm{H}-{ }^{1} \mathrm{H},{ }^{1} \mathrm{H}-{ }^{13} \mathrm{C}$ COSY spectra were recorded with a Bruker ARX 400 spectrometer for solutions in $\mathrm{CDCl}_{3}$ or $\mathrm{CD}_{3} \mathrm{OD}$. Chemical shifts are given in ppm downfield from internal $\mathrm{Me}_{4} \mathrm{Si}$. Mass spectra were measured using a MALTI-TOF-MS with $\alpha$-cyano4-hydroxycinnamic acid (CCA) as matrix or recorded with a VG PLATFORM mass spectrometer using the ESI technique to introduce the sample. Thin-layer chromatography (TLC) was performed on silica gel $\mathrm{HF}_{254}$ with detection by charring with $30 \%(\mathrm{v} / \mathrm{v}) \mathrm{H}_{2} \mathrm{SO}_{4}$ in $\mathrm{MeOH}$ or in some cases by UV detector. Column chromatography was conducted by elution of a column $(10 \times 240 \mathrm{~mm}, 18 \times 300 \mathrm{~mm}, 35 \times 400 \mathrm{~mm})$ of silica gel (100-200 mesh) with EtOAc-petroleum ether $\left(60-90{ }^{\circ} \mathrm{C}\right)$ as the eluent. Solutions were concentrated at $<60{ }^{\circ} \mathrm{C}$ under reduced pressure.

\section{2-O-Acetyl-3,4,6-tri- $O$-benzoyl- $\alpha$-D-glucopyranosyl trichloro- acetimidate 2}

To a solution of compound $\mathbf{1 a}(5.4 \mathrm{~g}, 22.9 \mathrm{mmol})$ in pyridine $(20 \mathrm{~mL})$ was added premixed $\mathrm{BzCl}-\mathrm{Pyr}(3.2 \mathrm{~mL}$ of $\mathrm{BzCl}$ in 3 $\mathrm{mL}$ of pyridine) mixture under $0{ }^{\circ} \mathrm{C}$. The reaction mixture was stirred at room temperature overnight, then poured into cold water and extracted with $\mathrm{CH}_{2} \mathrm{Cl}_{2}(3 \times 100 \mathrm{~mL})$. The organic phase was combined, dried over anhydrous $\mathrm{Na}_{2} \mathrm{SO}_{4}$, and concentrated. The residue generated above was subjected to hydrolysis using $95 \%$ aq. acetic acid at room temperature for $15 \mathrm{~min}$. The reaction mixture was poured into ice-water $(50 \mathrm{~mL})$ and extracted with $\mathrm{CH}_{2} \mathrm{Cl}_{2}(3 \times 30 \mathrm{~mL})$. The combined organic extracts were successively washed with saturated aq. $\mathrm{NaHCO}_{3}$ and water, dried over $\mathrm{MgSO}_{4}$, and filtered. The filtrate was evaporated and the residue was subsequently dissolved in anhydrous $\mathrm{CH}_{2} \mathrm{Cl}_{2}(15 \mathrm{~mL})$. To the above solution were added trichloroacetonitrile $(6 \mathrm{~mL}, 60 \mathrm{mmol})$ and DBU $(0.6 \mathrm{~mL})$ and the mixture was stirred at room temperature for 3 hours, then evaporated, and the residue was purified on a silica gel column (petroleum ether-EtOAc, $1.5: 1)$ to give $2(13.2 \mathrm{~g}, 85 \%$ from 1a) as a white foam; $[\alpha]_{\mathrm{D}}^{25}+32.3\left(c 1, \mathrm{CHCl}_{3}\right) ;{ }^{1} \mathrm{H}$ NMR $\left(\mathrm{CDCl}_{3}\right) \delta 1.96\left(\mathrm{~s}, 3 \mathrm{H}, \mathrm{CH}_{3} \mathrm{CO}\right), 4.45\left(\mathrm{dd}, 1 \mathrm{H}, J_{5,6 \mathrm{a}} 5.1, J_{6 \mathrm{a}, 6 \mathrm{~b}}\right.$ $\left.12.3 \mathrm{~Hz}, \mathrm{H}^{\mathrm{a}}-6\right), 4.55-4.63\left(\mathrm{~m}, 2 \mathrm{H}, \mathrm{H}-5, \mathrm{H}^{\mathrm{b}}-6\right), 5.43(\mathrm{dd}, 1 \mathrm{H}$, $\left.J_{2,3} 10.0 \mathrm{~Hz}, \mathrm{H}-2\right), 5.72\left(\mathrm{t}, 1 \mathrm{H}, J_{3,4}=J_{4,5}=10.0 \mathrm{~Hz}, \mathrm{H}-4\right)$, $6.08(\mathrm{t}, 1 \mathrm{H}, \mathrm{H}-3), 6.69$ (d, $\left.1 \mathrm{H}, J_{1,2} 3.7 \mathrm{~Hz}, \mathrm{H}-1\right), 7.26-8.02$
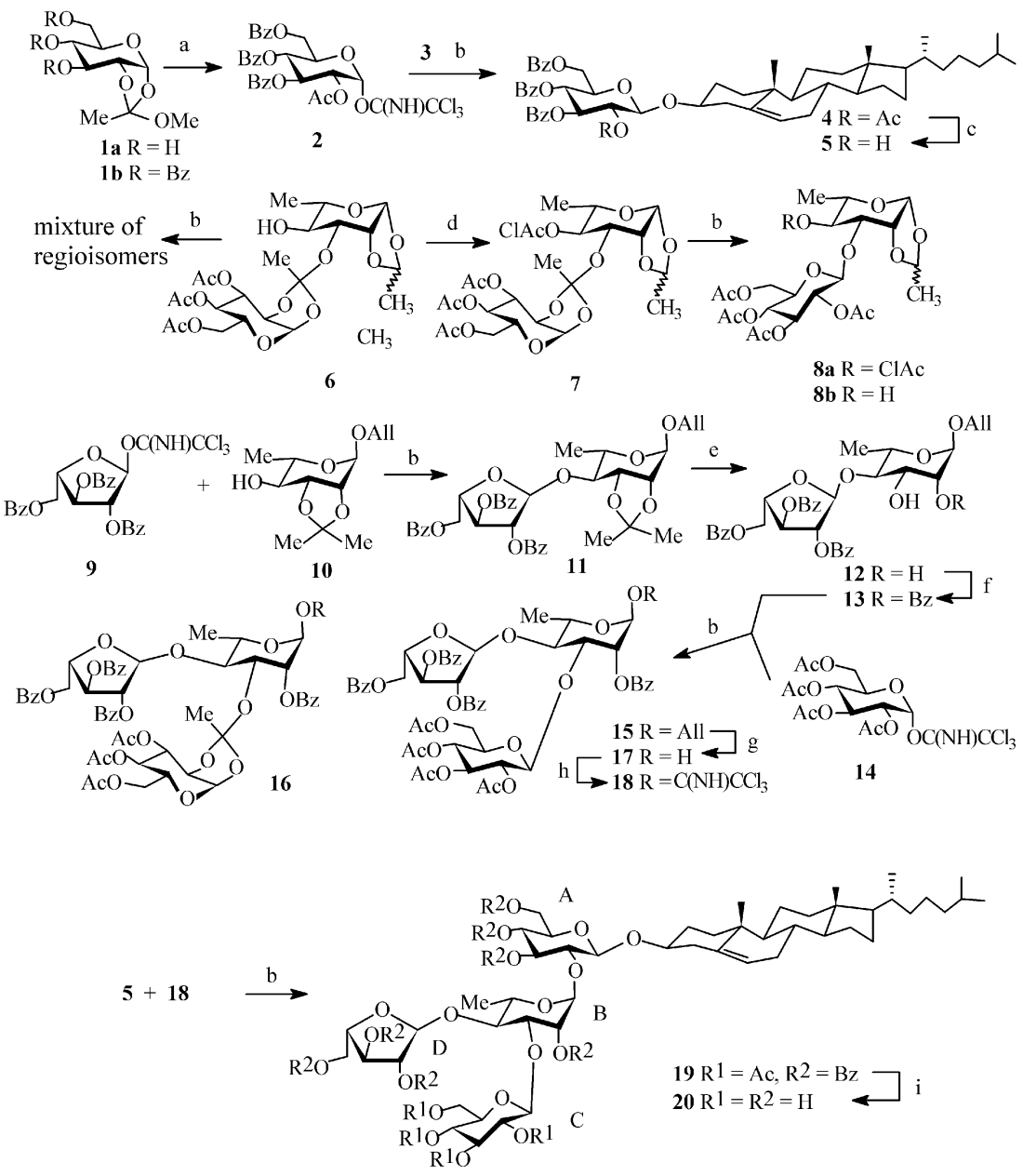

Scheme 1 Reagents and conditions (yields): a) $95 \% \mathrm{AcOH}, \mathrm{Cl}_{3} \mathrm{CCN}, \mathrm{DBU}, \mathrm{CH}_{2} \mathrm{Cl}_{2}(87 \%)$; b) TMSOTS, $\mathrm{CH}_{2} \mathrm{Cl}_{2}(91 \%$ for $\mathbf{4}, 77 \%$ for $\mathbf{8 a}, 83 \%$ for $\mathbf{1 1}$, $78 \%$ for 15, $86 \%$ for 19); c) $3 \%$ acetyl chloride in $\mathrm{MeOH}-\mathrm{CH}_{2} \mathrm{Cl}_{2}(1: 1),(89 \%)$; d) $\left(\mathrm{ClCH}_{2} \mathrm{CO}\right)_{2} \mathrm{O}, \mathrm{Pyr}(90 \%)$; e) $90 \% \mathrm{TFA}(88 \%)$; f) $\mathrm{BzCl}, \mathrm{Pyr}(79 \%)$; g) $\mathrm{PdCl}_{2}, \mathrm{NaOAc}, \mathrm{HOAc}$; ) $\mathrm{Cl}_{3} \mathrm{CCN}, \mathrm{DBU}, \mathrm{CH}_{2} \mathrm{Cl}_{2}$ (55\% for two steps); i) $\mathrm{NaOMe}, \mathrm{MeOH}(95 \%)$. 
$(\mathrm{m}, 15 \mathrm{H}, \mathrm{Ph}), 8.72(\mathrm{~s}, 1 \mathrm{H}, \mathrm{C}=\mathrm{NH})$ [MALDI-TOF-MS Calc. for $\mathrm{C}_{31} \mathrm{H}_{26} \mathrm{Cl}_{3} \mathrm{NNaO}_{10}(M+\mathrm{Na})^{+}:$700.05. Found: $m / z$, $\left.700(\mathrm{M}+\mathrm{Na})^{+}\right]$.

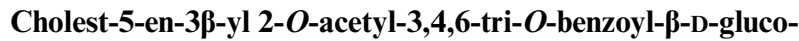 pyranoside 4}

Compound 2 (5.9 g, $8.7 \mathrm{mmol})$ and cholesterol 3 (3.32 g, $8.6 \mathrm{mmol})$ were dissolved in anhydrous dichloromethane $(40 \mathrm{~mL})$ at room temperature, and then TMSOTf $(156 \mu \mathrm{L}$, $0.86 \mathrm{mmol}$ ) was added dropwise. The mixture was stirred under these conditions for 4 hours, at the end of which time TLC showed that the donor $\mathbf{2}$ was completely consumed. The reaction mixture was quenched with $\mathrm{Et}_{3} \mathrm{~N}$ (4 drops) and the mixture were evaporated in vacuo to give a residue, which was purified by silica gel column chromatography (petroleum etherEtOAc, $1.5: 1)$ to give 4 as a syrup $(7.05 \mathrm{~g}, 91 \%)$; $[\alpha]_{\mathrm{D}}^{25}-15(c 1$, $\left.\mathrm{CHCl}_{3}\right) ;{ }^{1} \mathrm{H}$ NMR $\left(\mathrm{CDCl}_{3}\right) \delta 0.68-2.27\left(\mathrm{~m}, \mathrm{CH}_{3} \mathrm{CO}\right.$ and protons of cholesterol), $3.52(\mathrm{~m}, 1 \mathrm{H}, \mathrm{OCH}), 4.06(\mathrm{~m}, 1 \mathrm{H}, \mathrm{H}-5)$, $4.47\left(\mathrm{dd}, 1 \mathrm{H}, J_{5,6 \mathrm{a}} 6.0, J_{6 \mathrm{a}, 6 \mathrm{~b}} 12.0 \mathrm{~Hz}, \mathrm{H}^{\mathrm{a}}-6\right), 4.56(\mathrm{dd}, 1 \mathrm{H}$, $\left.J_{5,6 \mathrm{~b}} 3.2 \mathrm{~Hz}, \mathrm{H}^{\mathrm{b}}-6\right), 4.79\left(\mathrm{~d}, 1 \mathrm{H}, J_{1,2} 7.9 \mathrm{~Hz}, \mathrm{H}-1\right), 5.23(\mathrm{dd}, 1 \mathrm{H}$ $\left.J_{2,3} 9.7 \mathrm{~Hz}, \mathrm{H}-2\right), 5.35$ (br d, $\left.1 \mathrm{H},=\mathrm{CH}\right), 5.55\left(\mathrm{t}, 1 \mathrm{H}, J_{4,5} 9.7 \mathrm{~Hz}\right.$ $\mathrm{H}-4), 5.71\left(\mathrm{t}, 1 \mathrm{H}, J_{3,4} 9.7 \mathrm{~Hz}, \mathrm{H}-3\right), 7.30-8.00(\mathrm{~m}, 15 \mathrm{H}, \mathrm{Ph})$ [ESIMS Calc. for $\mathrm{C}_{56} \mathrm{H}_{70} \mathrm{NaO}_{10}(M+\mathrm{Na})^{+}$: 925.5. Found: $\left.m / z, 925.6(M+\mathrm{Na})^{+}\right]$.

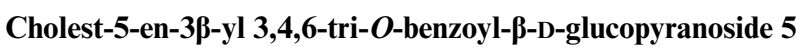

To a solution of $4(3.16 \mathrm{~g}, 3.5 \mathrm{mmol})$ in anhydrous $\mathrm{CH}_{2} \mathrm{Cl}_{2}-$ $\mathrm{CH}_{3} \mathrm{OH}(1: 1,50 \mathrm{~mL})$ was added acetyl chloride $(1.5 \mathrm{~mL})$ at $0{ }^{\circ} \mathrm{C}$. The mixture was sealed in a flask and stirred at room temperature. The reaction was detected by TLC until the starting material disappeared ( $c a .24$ hours), then the mixture was neutralized with pyridine, and concentrated to near dryness. The residue was passed through a short silica gel column to give $5(2.68 \mathrm{~g}, 89 \%)$ as a white solid; $[\alpha]_{\mathrm{D}}^{25}-6\left(c 1.8, \mathrm{CHCl}_{3}\right)$; ${ }^{1} \mathrm{H}$ NMR $\left(\mathrm{CDCl}_{3}\right) \delta 0.67-2.49$ (m, protons of cholesterol), $3.57-3.63(\mathrm{~m}, 1 \mathrm{H}, \mathrm{OCH}), 3.79\left(\mathrm{dd}, 1 \mathrm{H}, J_{1,2} 7.8, J_{2,3} 9.3 \mathrm{~Hz}\right.$, H-2), 4.03-4.08 (m, $1 \mathrm{H}, \mathrm{H}-5), 4.48$ (dd, $1 \mathrm{H}, J_{5,6 \mathrm{a}} 6.2, J_{6 \mathrm{a}, 6 \mathrm{~b}} 12.0$ $\mathrm{Hz}, \mathrm{H}-6), 4.55$ (dd, $\left.1 \mathrm{H}, J_{5,6 \mathrm{~b}} 3.5 \mathrm{~Hz}, \mathrm{H}-6\right), 4.66$ (d, $1 \mathrm{H}, J_{1,2} 7.8$ $\mathrm{Hz}, \mathrm{H}-1), 5.35$ (br d, $1 \mathrm{H},=\mathrm{CH}), 5.52$ (t, $\left.1 \mathrm{H}, J_{4,5} 9.6 \mathrm{~Hz}, \mathrm{H}-4\right)$, $5.71\left(\mathrm{t}, 1 \mathrm{H}, J_{3,4} 9.6 \mathrm{~Hz}, \mathrm{H}-3\right), 7.30-8.00(\mathrm{~m}, 15 \mathrm{H}, \mathrm{Ph})$ [ESIMS Calc. for $\mathrm{C}_{54} \mathrm{H}_{68} \mathrm{NaO}_{9}(M+\mathrm{Na})^{+}$]: 883.5. Found: $\mathrm{m} / z, 883$ $\left.(\mathrm{M}+\mathrm{Na})^{+}\right]\left(\right.$Calc. for $\mathrm{C}_{54} \mathrm{H}_{68} \mathrm{O}_{9}: \mathrm{C}, 75.35 ; \mathrm{H}, 7.91$. Found: $\mathrm{C}$, $75.68 ; \mathrm{H}, 7.70 \%)$.

\section{$3^{\prime}, 4^{\prime}, 6^{\prime}$-Tri- $O$-acetyl- $\alpha$-D-glucopyranose-1' $\mathbf{2}^{\prime}$-diyl 4- $O$-chloro- acetyl-1,2- $O-((R, S)$-ethylidene $)-\beta$-L-rhamnopyranos-3-yl orthoacetate 7}

To a solution of $6(790 \mathrm{mg}, 1.5 \mathrm{mmol})$ in pyridine $(3 \mathrm{~mL})$ was added chloroacetic anhydride $(342 \mathrm{mg}, 2 \mathrm{mmol})$. The mixture was kept at room temperature for 3 hours, then poured into cold water $(6 \mathrm{~mL})$, and extracted with $\mathrm{CH}_{2} \mathrm{Cl}_{2}(2 \times 30 \mathrm{~mL})$. The combined organic phase was dried over anhydrous sodium sulfate. After filtration and concentration under reduced pressure, the obtained residue was purified by column chromatography $(1: 1.5 \mathrm{EtOAc}-$ petroleum ether) to give $7(815 \mathrm{mg}$, $90 \%$ ). A small amount of pure $R$-isomer of 7 was obtained as crystals: mp $174-176{ }^{\circ} \mathrm{C}$; $[\alpha]_{\mathrm{D}}^{25}-65\left(c 1.1, \mathrm{CHCl}_{3}\right)$; ${ }^{1} \mathrm{H}$ NMR $\left(\mathrm{CDCl}_{3}\right) \delta 1.24\left(\mathrm{~d}, 3 \mathrm{H}, J_{5,6} 6.3 \mathrm{~Hz}, \mathrm{H}_{3}-6\right), 1.52(\mathrm{~d}, 3 \mathrm{H}, J 4.9 \mathrm{~Hz}$, $\left.\mathrm{CH}_{3} \mathrm{CH}\right), 1.77\left(\mathrm{~s}, 3 \mathrm{H}, \mathrm{CH}_{3}\right), 2.09,2.10,2.13(3 \mathrm{~s}, 9 \mathrm{H}, 3 \times \mathrm{Ac})$, 3.52 (dq, $\left.1 \mathrm{H}, J_{4,5} 9.4 \mathrm{~Hz}, \mathrm{H}-5\right), 3.90$ (m, $\left.1 \mathrm{H}, \mathrm{H}-5^{\prime}\right), 4.00$ (dd, $\left.1 \mathrm{H}, J_{2,3} 4.3, J_{3,4} 9.4 \mathrm{~Hz}, \mathrm{H}-3\right), 4.08,4.14(2 \mathrm{~d}, 2 \mathrm{H}, J 14.7 \mathrm{~Hz}$, $\left.\mathrm{ClCH}_{2}\right), 4.21-4.26\left(\mathrm{~m}, 2 \mathrm{H}, \mathrm{H}_{2}-6^{\prime}\right), 4.24(\mathrm{dd}, 1 \mathrm{H}, \mathrm{H}-2), 4.44$ $\left(\mathrm{dd}, 1 \mathrm{H}, \mathrm{H}-2^{\prime}\right), 4.94\left(\mathrm{t}, 1 \mathrm{H}, J_{3^{\prime}, 4^{\prime}}=J_{4^{\prime}, 5^{\prime}}=9.9 \mathrm{~Hz}, \mathrm{H}-4^{\prime}\right), 5.03(\mathrm{t}$, $\left.1 \mathrm{H}, J_{3,4}=J_{4,5}=9.2 \mathrm{~Hz}, \mathrm{H}-4\right), 5.18\left(\mathrm{dd}, 1 \mathrm{H}, J_{2^{\prime}, 3^{\prime}} 3.2 \mathrm{~Hz}, \mathrm{H}-3^{\prime}\right)$, 5.25 (d, $\left.1 \mathrm{H}, J_{1,2} 2.2 \mathrm{~Hz}, \mathrm{H}-1\right), 5.34\left(\mathrm{q}, 1 \mathrm{H}, \mathrm{CH}_{3} \mathrm{CH}\right), 5.70$ (d, $1 \mathrm{H}, J_{1^{\prime}, 2^{\prime}} 5.2 \mathrm{~Hz}, \mathrm{H}-1^{\prime}$ ) (Calc. for $\mathrm{C}_{24} \mathrm{H}_{33} \mathrm{ClO}_{15}$ : C, 48.28; H, 5.53. Found: C, 48.46; H, 5.41\%).

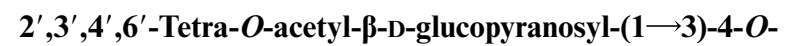
chloroacetyl-1,2- $O-((R, S)$-ethylidene $)-\beta$-L-rhamnopyranose $8 \mathrm{a}$

A suspension of 7 (620 mg, $1.04 \mathrm{mmol})$ containing $4 \AA$ Molecular Sieves in anhydrous $\mathrm{CH}_{2} \mathrm{Cl}_{2}(20 \mathrm{~mL})$ was cooled to $-10{ }^{\circ} \mathrm{C}$, then TMSOTf $(10 \mu \mathrm{L}, 0.05 \mathrm{mmol})$ was added under $\mathrm{N}_{2}$ flow. The mixture was stirred at this temperature for about 1 hour, then neutralized with 2 drops of triethylamine. After filtration and concentration under reduced pressure, the obtained residue was purified by column chromatography (1: 1.5 EtOAc-petroleum ether) to give $\mathbf{8 a}$ as crystals $(477 \mathrm{mg}$, $77 \%)$. Pure $R$-isomer gave the following physical data: $\mathrm{mp}$ $158-160{ }^{\circ} \mathrm{C} ;[a]_{\mathrm{D}}^{25}-35\left(c 1.4, \mathrm{CHCl}_{3}\right) ;{ }^{1} \mathrm{H}$ NMR $\left(\mathrm{CDCl}_{3}\right) \delta 1.22$ (d, $\left.3 \mathrm{H}, J_{5,6} 6.2 \mathrm{~Hz}, \mathrm{H}_{3}-6\right), 1.53\left(\mathrm{~d}, 3 \mathrm{H}, J 4.9 \mathrm{~Hz}, \mathrm{CH}_{3} \mathrm{CH}\right)$, 2.00, 2.03, 2.05, $2.10(4 \mathrm{~s}, 12 \mathrm{H}, 4 \times \mathrm{Ac}), 3.49\left(\mathrm{dq}, 1 \mathrm{H}, J_{4,5}\right.$ $\left.9.5 \mathrm{~Hz}, \mathrm{H}-5^{\prime}\right), 3.70\left(\mathrm{~m}, 1 \mathrm{H}, \mathrm{H}-5^{\prime}\right), 4.00\left(\mathrm{dd}, 1 \mathrm{H}, J_{2,3} 4.0\right.$, $\left.J_{3,4} 9.7 \mathrm{~Hz}, \mathrm{H}-3\right), 4.10,4.14\left(2 \mathrm{~d}, 2 \mathrm{H}, J 15 \mathrm{~Hz}, \mathrm{ClCH}_{2}\right), 4.21-$ $4.24\left(\mathrm{~m}, 3 \mathrm{H}, \mathrm{H}-2, \mathrm{H}_{2}-6^{\prime}\right), 4.78\left(\mathrm{~d}, 1 \mathrm{H}, J_{1^{\prime}, 2^{\prime}} 8.0 \mathrm{~Hz}, \mathrm{H}-1^{\prime}\right), 5.04$ $\left(\mathrm{t}, 1 \mathrm{H}, \mathrm{H}-2^{\prime}\right), 5.11\left(\mathrm{t}, 1 \mathrm{H}, J_{3^{\prime}, 4^{\prime}}=J_{4^{\prime}, 5^{\prime}}=9.5 \mathrm{~Hz}, \mathrm{H}-4^{\prime}\right), 5.03$ $\left(\mathrm{t}, 1 \mathrm{H}, J_{3,4}=J_{4,5}=9.7 \mathrm{~Hz}, \mathrm{H}-4\right), 5.18\left(\mathrm{t}, 1 \mathrm{H}, J_{2^{\prime}, 3^{\prime}} 9.5 \mathrm{~Hz}\right.$, $\left.\mathrm{H}^{\prime} 3^{\prime}\right), 5.25\left(\mathrm{~d}, 1 \mathrm{H}, J_{1,2} 2.0 \mathrm{~Hz}, \mathrm{H}-1\right), 5.33\left(\mathrm{q}, 1 \mathrm{H}, \mathrm{CH}_{3} \mathrm{CH}\right)$ (Calc. for $\mathrm{C}_{24} \mathrm{H}_{33} \mathrm{ClO}_{15}$ : C, 48.28; H, 5.53. Found: C, 48.37; $\mathrm{H}, 5.43 \%)$.

Allyl 2',3',5' -tri- $O$-benzoyl- $\alpha$-L-arabinofuranosyl-(1 $\longrightarrow 4)$-2,3-Oisopropylidene- $\alpha$-L-rhamnopyranoside 11

To an ice-water-cooled solution of $\mathbf{9}(6.67 \mathrm{~g}, 11 \mathrm{mmol})$ and $\mathbf{1 0}$ $(2.45 \mathrm{~g}, 10 \mathrm{mmol})$ in anhydrous $\mathrm{CH}_{2} \mathrm{Cl}_{2}(50 \mathrm{~mL})$ was added TMSOTf $(70 \mu \mathrm{L}, 0.39 \mathrm{mmol})$. The mixture was stirred at this temperature for 2 hours, then quenched with $\mathrm{Et}_{3} \mathrm{~N}$ (4 drops). The mixture was evaporated in vacuo to give a residue, which was purified on a silica gel column chromatography (petroleum ether-EtOAc, $2: 1)$ to give disaccharide $11(5.73 \mathrm{~g}, 83 \%)$ as a syrup; $[\alpha]_{\mathrm{D}}^{25}-11\left(c 1, \mathrm{CHCl}_{3}\right) ;{ }^{1} \mathrm{H}$ NMR $\left(\mathrm{CDCl}_{3}\right) \delta 1.30(\mathrm{~s}, 3 \mathrm{H}$, $\left.\mathrm{CH}_{3}\right), 1.31\left(\mathrm{~d}, 3 \mathrm{H}, \mathrm{H}_{3}-6\right), 1.55\left(\mathrm{~s}, 3 \mathrm{H}, \mathrm{CH}_{3}\right), 3.69$ (dd, $1 \mathrm{H}$, J 7.2, $9.9 \mathrm{~Hz}, \mathrm{H}-4), 3.76$ (dq, $1 \mathrm{H}, \mathrm{H}-5), 4.00-4.21$ (m, $3 \mathrm{H}, \mathrm{H}-2$, $\left.\mathrm{CH}_{2}=\mathrm{CHCH}_{2}\right), 4.33(\mathrm{dd}, 1 \mathrm{H}, J 7.0,5.8 \mathrm{~Hz}, \mathrm{H}-3), 4.59(\mathrm{~m}, 1 \mathrm{H}$, H-4'), 4.7 (dd, $\left.1 \mathrm{H}, J 5.6,11.8 \mathrm{~Hz}, \mathrm{H}^{\mathrm{a}}-5^{\prime}\right), 4.8$ (dd, $1 \mathrm{H}, J 3.6 \mathrm{~Hz}$, $\left.\mathrm{H}^{\mathrm{b}}-5^{\prime}\right), 5.04$ (s, $\left.1 \mathrm{H}, \mathrm{H}-1\right), 5.20-5.35\left(\mathrm{~m}, 2 \mathrm{H}, \mathrm{CH}_{2}=\mathrm{CHCH}_{2}\right.$ ), $5.54\left(\mathrm{~d}, 1 \mathrm{H}, J 4.6 \mathrm{~Hz}, \mathrm{H}-3^{\prime}\right), 5.60\left(\mathrm{~s}, 1 \mathrm{H}, \mathrm{H}-2^{\prime} / \mathrm{H}^{-1}{ }^{\prime}\right), 5.81$ (s, $\left.1 \mathrm{H}, \mathrm{H}-1^{\prime} / \mathrm{H}-2^{\prime}\right), 5.87-5.95\left(\mathrm{~m}, 1 \mathrm{H}, \mathrm{CH}_{2}=\mathrm{CHCH}_{2}\right), 7.28-8.09$ $(\mathrm{m}, 15 \mathrm{H}, \mathrm{Ph})\left(\right.$ Calc. for $\mathrm{C}_{38} \mathrm{H}_{40} \mathrm{O}_{12}: \mathrm{C}, 66.28 ; \mathrm{H}, 5.81$. Found: C, 66.04; H, 6.09\%).

\section{Allyl 2',3',5'-tri- $O$-benzoyl- $\alpha$-L-arabinofuranosyl-(1 $\longrightarrow 4)-2-O$ - benzoyl- $\alpha$-L-rhamnopyranoside 13}

To a solution of compound 11 (4.79 g, $6.96 \mathrm{mmol})$ in $\mathrm{CH}_{2} \mathrm{Cl}_{2}$ $(2 \mathrm{~mL})$ was added $90 \%$ aq. trifluoacetic acid $(10 \mathrm{~mL})$. The mixture was stirred at room temperature for $20 \mathrm{~min}$, then the mixture was evaporated in vacuo in the presence of toluene $(2 \times 15 \mathrm{~mL})$ and the residue was purified on a short silica gel column (petroleum ether-EtOAc, $1: 1$ ) to give crude diol 12 as a syrup.

To an ice-water-cooled solution of $12(3.1 \mathrm{~g}, 4.78 \mathrm{mmol})$ in pyridine $(20 \mathrm{~mL})$ was added benzoyl chloride $(0.58 \mathrm{~mL}$, $5.02 \mathrm{mmol}$ ). The mixture was stirred at this temperature for 6 hours, then poured into water and extracted with $\mathrm{CH}_{2} \mathrm{Cl}_{2}$ $(2 \times 75 \mathrm{~mL})$. The combined organic phase was dried over $\mathrm{Na}_{2} \mathrm{SO}_{4}$ and evaporated to give a syrup. Column chromatography gave pure $\mathbf{1 3}$ as an amorphous solid (3.7 g, 79\%); $[a]_{\mathrm{D}}^{25}+13\left(c 1, \mathrm{CHCl}_{3}\right) ;{ }^{1} \mathrm{H} \mathrm{NMR}\left(\mathrm{CDCl}_{3}\right) \delta 1.42(\mathrm{~d}, 3 \mathrm{H}, J 6.1$ $\left.\mathrm{Hz}, \mathrm{H}_{3}-6\right), 3.79$ (t, $\left.1 \mathrm{H}, J 9.3 \mathrm{~Hz}, \mathrm{H}-4\right), 3.87$ (dq, $\left.1 \mathrm{H}, \mathrm{H}-5\right)$, 4.01-4.23 (m, $\left.2 \mathrm{H}, \mathrm{CH}_{2}=\mathrm{CHCH}_{2}\right), 4.39\left(\mathrm{dd}, 1 \mathrm{H}, J_{2,3} 3.3 \mathrm{~Hz}\right.$, $\mathrm{H}-3), 4.65-4.71\left(\mathrm{~m}, 2 \mathrm{H}, \mathrm{H}-4^{\prime}\right.$ and $\left.\mathrm{H}^{\mathrm{a}}-5^{\prime}\right), 4.80(\mathrm{dd}, 1 \mathrm{H}, J 8.4$ $\left.\mathrm{Hz}, \mathrm{H}^{\mathrm{b}}-5^{\prime}\right), 4.92(\mathrm{~d}, 1 \mathrm{H}, J 1.4 \mathrm{~Hz}, \mathrm{H}-1), 5.21-5.36$ (m, $2 \mathrm{H}$, $\left.\mathrm{CH}_{2}=\mathrm{CHCH}_{2}\right), 5.36(\mathrm{dd}, 1 \mathrm{H}, \mathrm{H}-2), 5.43(\mathrm{~d}, 1 \mathrm{H}, J 2.7 \mathrm{~Hz}$, $\left.\mathrm{H}-2^{\prime}\right)$, 5.68-5.71 (m, $2 \mathrm{H}, \mathrm{H}-1^{\prime}$ and $\left.\mathrm{H}-3^{\prime}\right)$, 5.9-6.00 (m, $1 \mathrm{H}$, $\left.\mathrm{CH}_{2}=\mathrm{CHCH}_{2}\right), 7.25-8.09(\mathrm{~m}, 20 \mathrm{H}, \mathrm{Ph})$ [ESIMS calcd for $\mathrm{C}_{42} \mathrm{H}_{40} \mathrm{NaO}_{13}(M+\mathrm{Na})^{+}$: 775.2. Found: $\left.m / z, 775(\mathrm{M}+\mathrm{Na})^{+}\right]$ 
(Calc. for $\mathrm{C}_{42} \mathrm{H}_{40} \mathrm{O}_{13}$ : C, 67.02; H, 5.32. Found: C, 66.90; $\mathrm{H}, 5.44 \%)$.

\section{Allyl $2^{\mathrm{D}}, 3^{\mathrm{D}}, 5^{\mathrm{D}}$-tri- $O$-benzoyl- $\alpha$-L-arabinofuranosyl-(1 $\left.\longrightarrow 4\right)$ - $\left[2^{\mathrm{C}}, 3^{\mathrm{C}}, 4^{\mathrm{C}}, 6^{\mathrm{C}}\right.$-tetra- $O$-acetyl- $\beta$-D-glucopyranosyl-(1 $\left.\left.\longrightarrow 3\right)\right]-2^{\mathrm{B}}-O$ - benzoyl- $\alpha$-L-rhamnopyranoside 15}

To a cooled solution $\left(0^{\circ} \mathrm{C}\right)$ of $\mathbf{1 3}(2.106 \mathrm{~g}, 2.8 \mathrm{mmol})$ and $\mathbf{1 4}$ (1.45 g, $2.94 \mathrm{mmol})$ in anhydrous $\mathrm{CH}_{2} \mathrm{Cl}_{2}(25 \mathrm{~mL})$ was added TMSOTf $(50 \mu \mathrm{L}, 0.28 \mathrm{mmol})$. The mixture was stirred at this temperature for $40 \mathrm{~min}$, then another portion of TMSOTf $(15 \mu \mathrm{L}, 0.08 \mathrm{mmol})$ was added. The mixture was stirred at $0{ }^{\circ} \mathrm{C}$ for 3 hours, then was quenched with $\mathrm{Et}_{3} \mathrm{~N}$ (4 drops). The mixture was evaporated in vacuo to give a residue, which was purified by silica gel column chromatography (petroleum ether-EtOAc, $1: 1)$ to give trisaccharide $15(2.36 \mathrm{~g}, 78 \%)$ as a syrup; $[\alpha]_{\mathrm{D}}^{25}-1\left(c 1, \mathrm{CHCl}_{3}\right) ;{ }^{1} \mathrm{H}$ NMR $\left(\mathrm{CDCl}_{3}\right) \delta 1.37(\mathrm{~d}, 3 \mathrm{H}$, $\left.J 6.4 \mathrm{~Hz}, \mathrm{H}_{3}-6^{\mathrm{B}}\right), 1.49,1.82(2 \mathrm{~s}, 2 \times 3 \mathrm{H}, 2 \mathrm{Ac}), 2.04(\mathrm{~s}, 6 \mathrm{H}$, $2 \mathrm{Ac}), 3.84-3.90\left(\mathrm{~m}, 2 \mathrm{H}, \mathrm{H}-5^{\mathrm{B}}, \mathrm{H}-5^{\mathrm{C}}\right), 4.00(\mathrm{t}, 1 \mathrm{H}, J 9.4 \mathrm{~Hz}$, $\left.\mathrm{H}-4^{\mathrm{B}}\right), 4.05\left(\mathrm{~m}, 1 \mathrm{H}\right.$, one proton of $\left.\mathrm{CH}_{2}=\mathrm{CHCH}_{2}\right), 4.14-4.20$ $\left(\mathrm{m}, 3 \mathrm{H}, \mathrm{H}_{2}-6^{\mathrm{C}}\right.$, one proton of $\left.\mathrm{CH}_{2}=\mathrm{CHCH}_{2}\right), 4.42(\mathrm{dd}, 1 \mathrm{H}$, $\left.J 3.4, J 10.4 \mathrm{~Hz}, \mathrm{H}-3^{\mathrm{B}}\right), 4.65-4.70\left(\mathrm{~m}, 2 \mathrm{H}, \mathrm{H}-4^{\mathrm{D}}, \mathrm{H}-1^{\mathrm{C}}\right), 4.80$ $5.02\left(\mathrm{~m}, 5 \mathrm{H}, J 7.8, J 9.1, J 1.5 \mathrm{~Hz}, \mathrm{H}_{2}-5^{\mathrm{D}}, \mathrm{H}-1^{\mathrm{B}}, \mathrm{H}-2^{\mathrm{C}}, \mathrm{H}-4^{\mathrm{C}}\right)$, $5.21\left(\mathrm{t}, 1 \mathrm{H}, J 9.3 \mathrm{~Hz}, \mathrm{H}_{-}{ }^{\mathrm{C}}\right), 5.25\left(\mathrm{~m}, 1 \mathrm{H}, \mathrm{CH}_{2}=\mathrm{CHCH}_{2}\right), 5.31$ $\left(\mathrm{dd}, 1 \mathrm{H}, \mathrm{H}-2^{\mathrm{B}}\right), 5.34\left(\mathrm{~m}, 1 \mathrm{H}, \mathrm{CH}_{2}=\mathrm{CHCH}_{2}\right), 5.53(\mathrm{~d}, 1 \mathrm{H}, J 1.0$ $\left.\mathrm{Hz}, \mathrm{H}-1^{\mathrm{D}}\right), 5.65\left(\right.$ br d, $\left.1 \mathrm{H}, J 3.8 \mathrm{~Hz}, \mathrm{H}^{-} 3^{\mathrm{D}}\right), 5.77\left(\mathrm{~s}, 1 \mathrm{H}, \mathrm{H}-2^{\mathrm{D}}\right.$ ), $5.95\left(\mathrm{~m}, 1 \mathrm{H}, \mathrm{CH}_{2}=\mathrm{CHCH}_{2}\right), 7.26-8.11(\mathrm{~m}, 20 \mathrm{H}, \mathrm{Ph})(\mathrm{Calc}$. for $\mathrm{C}_{56} \mathrm{H}_{58} \mathrm{O}_{22}: \mathrm{C}, 62.11 ; \mathrm{H}, 5.36$. Found: $\mathrm{C}, 62.38 ; \mathrm{H}, 5.37 \%$ ).

The by-product was elucidated to be the orthoester 16; $[a]_{\mathrm{D}}^{25}-6\left(c 1, \mathrm{CHCl}_{3}\right) ;{ }^{1} \mathrm{H} \mathrm{NMR}\left(\mathrm{CDCl}_{3}\right) \delta 1.41(\mathrm{~d}, 3 \mathrm{H}, J 5.5 \mathrm{~Hz}$, $\left.\mathrm{H}_{3}-6\right), 1.75\left(\mathrm{~s}, 3 \mathrm{H}, \mathrm{CH}_{3} \mathrm{O}\right), 1.94,1.95,2.07(3 \mathrm{~s}, 3 \times 3 \mathrm{H}$, $3 \times \mathrm{Ac}), 3.77-3.79(\mathrm{~m}, 1 \mathrm{H}), 3.87-3.90(\mathrm{~m}, 2 \mathrm{H}), 4.07(\mathrm{dd}, 1 \mathrm{H})$, $4.11(\mathrm{~m}, 2 \mathrm{H}), 4.15(\mathrm{dd}, 1 \mathrm{H}), 4.18-4.23(\mathrm{~m}, 1 \mathrm{H}), 4.30(\mathrm{dd}$, $1 \mathrm{H}), 4.66-4.70(\mathrm{~m}, 2 \mathrm{H}), 4.74-4.78(\mathrm{~m}, 2 \mathrm{H}), 4.85$ (d, $1 \mathrm{H}), 4.99$ $(\mathrm{t}, 1 \mathrm{H}), 5.24-5.36(\mathrm{~m}, 2 \mathrm{H}), 5.48(\mathrm{dd}, 1 \mathrm{H}), 5.55(\mathrm{dd}, 1 \mathrm{H})$, $5.65(\mathrm{~d}, 1 \mathrm{H}), 5.71(\mathrm{~d}, 1 \mathrm{H}), 5.72(\mathrm{~s}, 1 \mathrm{H}), 5.90-5.99(\mathrm{~m}, 1 \mathrm{H})$, 7.25-8.10 (m, 20 H, Ph).

$2^{\mathrm{D}}, 3^{\mathrm{D}}, 5^{\mathrm{D}}$-Tri- $O$-benzoyl- $\alpha$-L-arabinofuranosyl- $(1 \longrightarrow 4)$ $\left[2^{\mathrm{C}}, 3^{\mathrm{C}}, 4^{\mathrm{C}}, 6^{\mathrm{C}}\right.$-tetra- $O$-acetyl- $\beta$-D-glucopyranosyl-( $\left.\left.1 \longrightarrow 3\right)\right]-2^{\mathrm{B}}-O$ benzoyl- $\alpha$-L-rhamnopyranosyl 2,2,2-trichloroacetimidate 18

To a solution of 15 (1.4 g, $1.29 \mathrm{mmol})$ in 90\% $\mathrm{AcOH}$ (aq., $10 \mathrm{~mL}$ ) were added $\mathrm{NaOAc}(497 \mathrm{mg}, 5.2 \mathrm{mmol})$ and $\mathrm{PdCl}_{2}$ (497 mg, $2.6 \mathrm{mmol}$ ). The mixture was stirred at room temperature for 16 hours, at the end of which time TLC $(1: 1$ petroleum ether-EtOAc) showed the completion of the reaction. The mixture was diluted with $\mathrm{CH}_{2} \mathrm{Cl}_{2}(30 \mathrm{~mL})$ and then filtered. The filtrate was neutralized with saturated aq. $\mathrm{NaHCO}_{3}$ and extracted with $\mathrm{CH}_{2} \mathrm{Cl}_{2}(2 \times 50 \mathrm{~mL})$. The organic phase was combined and concentrated, then subjected to silica gel column chromatography (1:1 petroleum ether-EtOAc). The product generated above (17) was dissolved in $\mathrm{CH}_{2} \mathrm{Cl}_{2}(20 \mathrm{~mL})$ and trichloroacetonitrile $(1.0 \mathrm{~mL}, 10 \mathrm{mmol})$, and DBU $(0.12 \mathrm{~mL})$ was added. The mixture was stirred at room temperature for 3 hours. TLC ( $2: 1$ petroleum ether-EtOAc) indicated that the reaction was complete. The mixture was concentrated under reduced pressure and then purified on a silica gel column using $2: 1$ petroleum ether-EtOAc as eluent to give $\mathbf{1 8}$ as a syrup (842 mg, 55\%); $[a]_{\mathrm{D}}^{25}-6\left(c 1, \mathrm{CHCl}_{3}\right) ;{ }^{1} \mathrm{H} \mathrm{NMR}\left(\mathrm{CDCl}_{3}\right) \delta 1.41$ $\left(\mathrm{d}, 3 \mathrm{H}, \mathrm{H}_{3}-6^{\mathrm{B}}\right), 1.44,1.83,2.02,2.04(4 \mathrm{~s}, 4 \times 3 \mathrm{H}, 4 \times \mathrm{Ac}), 3.84$ $\left(\mathrm{m}, 1 \mathrm{H}, \mathrm{H}-5^{\mathrm{B}}\right), 4.00-4.21\left(\mathrm{~m}, 4 \mathrm{H}, \mathrm{H}-5^{\mathrm{C}}, \mathrm{H}-4^{\mathrm{B}}, \mathrm{H}_{2}-6^{\mathrm{C}}\right), 4.49(\mathrm{dd}$, $\left.1 \mathrm{H}, J 3.5, J 8.9 \mathrm{~Hz}, \mathrm{H}-3^{\mathrm{B}}\right), 4.70-4.75\left(\mathrm{~m}, 2 \mathrm{H}, \mathrm{H}-1^{\mathrm{C}}, \mathrm{H}-4^{\mathrm{D}}\right)$, $4.80,4.90\left(2 \mathrm{dd}, 2 \mathrm{H}, J 7.9, J 9.7, J 6.0 \mathrm{~Hz}, \mathrm{H}_{2}-5^{\mathrm{D}}\right), 5.01-5.09$ $\left(\mathrm{m}, 2 \mathrm{H}, J 8.0, J 9.3 \mathrm{~Hz}, \mathrm{H}-2^{\mathrm{C}}, \mathrm{H}-4^{\mathrm{C}}\right), 5.25(\mathrm{t}, 1 \mathrm{H}, J 9.3 \mathrm{~Hz}$, $\left.\mathrm{H}-3^{\mathrm{C}}\right), 5.47\left(\mathrm{dd}, 1 \mathrm{H}, J 3.4, J 1.9 \mathrm{~Hz}, \mathrm{H}-2^{\mathrm{B}}\right), 5.58\left(\mathrm{~s}, 1 \mathrm{H}, \mathrm{H}^{-1}{ }^{\mathrm{D}}\right)$, $5.66\left(\mathrm{~d}, 1 \mathrm{H}, J 3.2 \mathrm{~Hz}, \mathrm{H}-3^{\mathrm{D}}\right), 5.73\left(\mathrm{~s}, 1 \mathrm{H}, \mathrm{H}-2^{\mathrm{D}}\right), 6.36(\mathrm{~d}, 1 \mathrm{H}$, $\left.J 1.6 \mathrm{~Hz}, \mathrm{H}-1^{\mathrm{B}}\right), 7.25-8.08(\mathrm{~m}, 20 \mathrm{H}, \mathrm{Ph}), 8.76(\mathrm{~s}, 1 \mathrm{H}, \mathrm{C}=\mathrm{NH})$ (Calc. for $\mathrm{C}_{55} \mathrm{H}_{54} \mathrm{Cl}_{3} \mathrm{NO}_{22}$ : C, 55.62; $\mathrm{H}, 4.55$. Found: $\mathrm{C}, 55.41$; $\mathrm{H}, 4.73 \%)$.

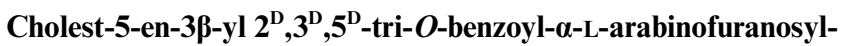
$(1 \longrightarrow 4)$-[2 $2^{\mathrm{C}}, 3^{\mathrm{C}}, 4^{\mathrm{C}}, 6^{\mathrm{C}}$-tetra- $O$-acetyl- $\beta$-D-glucopyranosyl-( $\left.\left.1 \longrightarrow 3\right)\right]$ $2^{\mathrm{B}}$ - $O$-benzoyl- $\alpha$-L-rhamnopyranosyl- $(1 \longrightarrow 2)-3^{\mathrm{A}}, 4^{\mathrm{A}}, 6^{\mathrm{A}}$-tri- $O$ benzoyl- $\beta$-D-glucopyranoside 19

Pre-dried compound 18 (1.15 g, $9.7 \mathrm{mmol})$ and glycosylated cholesterol derivative $5(874 \mathrm{mg}, 9.7 \mathrm{mmol})$ were dissolved in anhydrous $\mathrm{CH}_{2} \mathrm{Cl}_{2}(10 \mathrm{~mL})$ in an ice-water-bath, and then TMSOTf $(50 \mu \mathrm{L}, 0.28 \mathrm{mmol})$ was added. The mixture was stirred at this temperature for 2 hours, at the end of which time TLC showed that the donor $\mathbf{1 8}$ was completely consumed. The reaction mixture was quenched with $\mathrm{Et}_{3} \mathrm{~N}$ (2 drops) and the mixture was evaporated in vacuo to give a residue, which was purified by silica gel column chromatography (petroleum etherEtOAc, $1.5: 1)$ to give 19 as a syrup $(1.57 \mathrm{~g}, 86 \%) ;[\alpha]_{\mathrm{D}}^{25}+11(c$ $\left.1.2, \mathrm{CHCl}_{3}\right) ;{ }^{1} \mathrm{H} \mathrm{NMR}\left(\mathrm{CDCl}_{3}\right) \delta 1.62,1.81,1.88,1.98(4 \mathrm{~s}, 12$ $\mathrm{H}, 4 \times \mathrm{Ac}), 3.24\left(\mathrm{dt}, 1 \mathrm{H}, J 2.5,9.9 \mathrm{~Hz}, \mathrm{H}-5^{\mathrm{C}}\right), 3.59(\mathrm{~m}, 1 \mathrm{H}, \mathrm{H}-3$ of cholesterol), 3.90 (dd, $\left.1 \mathrm{H}, J 2.5,12.4 \mathrm{~Hz}, \mathrm{H}-6^{\mathrm{C}}\right), 3.95-4.05$ $\left(\mathrm{m}, 3 \mathrm{H}, \mathrm{H}-6^{\mathrm{C}}, \mathrm{H}-4^{\mathrm{B}}, \mathrm{H}-2^{\mathrm{A}}\right), 4.09\left(\mathrm{~m}, 1 \mathrm{H}, \mathrm{H}-5^{\mathrm{A}}\right), 4.20-4.30(\mathrm{~m}$, $\left.2 \mathrm{H}, \mathrm{H}-3^{\mathrm{B}}, \mathrm{H}-5^{\mathrm{B}}\right), 4.50\left(\mathrm{dd}, 1 \mathrm{H}, J 6.0,11.2 \mathrm{~Hz}, \mathrm{H}-6^{\mathrm{A}}\right), 4.61(\mathrm{dd}$, $\left.1 \mathrm{H}, J 7.9 \mathrm{~Hz}, \mathrm{H}-1^{\mathrm{C}}\right), 4.62-4.69\left(\mathrm{~m}, 2 \mathrm{H}, \mathrm{H}-4^{\mathrm{D}}, \mathrm{H}-5^{\mathrm{D}}\right), 4.74-4.77$ $\left(\mathrm{m}, 2 \mathrm{H}, J 7.8 \mathrm{~Hz}, \mathrm{H}-1^{\mathrm{A}}, \mathrm{H}-5^{\mathrm{D}}\right), 4.81(\mathrm{dd}, 1 \mathrm{H}, J 7.9,9.7 \mathrm{~Hz}$, $\left.\mathrm{H}-2^{\mathrm{C}}\right), 4.90\left(\mathrm{t}, 1 \mathrm{H}, \mathrm{H}-4^{\mathrm{C}}\right), 5.06\left(\mathrm{t}, 1 \mathrm{H}, \mathrm{H}-3^{\mathrm{C}}\right), 5.08$ (br s, $2 \mathrm{H}$, $\left.\mathrm{H}-1^{\mathrm{B}}, \mathrm{H}-2^{\mathrm{B}}\right), 5.32(\mathrm{~m}, 1 \mathrm{H},=\mathrm{CH}), 5.45\left(\right.$ br s, $\left.1 \mathrm{H}, \mathrm{H}^{-1}{ }^{\mathrm{D}}\right), 5.48$ (t, $\left.1 \mathrm{H}, J 9.5 \mathrm{~Hz}, \mathrm{H}-4^{\mathrm{A}}\right), 5.56$ (d, $\left.1 \mathrm{H}, J 3.9 \mathrm{~Hz}, \mathrm{H}-3^{\mathrm{D}}\right), 5.75$ (s, $\left.1 \mathrm{H}, \mathrm{H}-2^{\mathrm{D}}\right), 5.80\left(\mathrm{t}, 1 \mathrm{H}, \mathrm{H}-3^{\mathrm{A}}\right), 7.25-8.09(\mathrm{~m}, 35 \mathrm{H}, \mathrm{Ph}) ;{ }^{13} \mathrm{C}$ NMR $\left(\mathrm{CDCl}_{3} ; 100 \mathrm{MHz}\right)$ for sugar residues: $\delta_{\mathrm{C}} 18.3(\mathrm{C}-6$ of rhamnose), 60.9, 63.5, 63.9, 68.0, 70.1, 71.1, 71.2, 71.9, 72.8, $74.1,74.9,75.8,77.2,78.0,78.5,80.0,81.9,82.0$ (C-2-C-5), $97.7\left(\mathrm{C}-1^{\mathrm{A}}\right), 99.6\left(\mathrm{C}-1^{\mathrm{C}}\right), 100.3\left(\mathrm{C}-1^{\mathrm{B}}\right), 105.9\left(\mathrm{C}-1^{\mathrm{D}}\right), 165.2$, $165.4,165.5,165.6,165.7,166.0,166.1(7 \times \mathrm{PhCO}), 168.5$, $169.0,169.9,170.7\left(4 \times \mathrm{CH}_{3} \mathrm{CO}\right)$; ESIMS (positive) [calc. for $\mathrm{C}_{107} \mathrm{H}_{120} \mathrm{NaO}_{30}(M+\mathrm{Na})^{+}:$1907.8. Found: $m / z, 1908.2(\mathrm{M}+$ $\left.\mathrm{Na})^{+}\right]\left(\right.$Calc. for $\mathrm{C}_{107} \mathrm{H}_{120} \mathrm{O}_{30}: \mathrm{C}, 68.15 ; \mathrm{H}, 6.37$. Found: $\mathrm{C}, 68.01$; $\mathrm{H}, 6.44 \%)$.

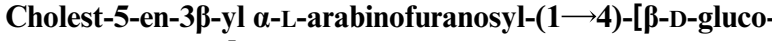 pyranosyl-( $1 \longrightarrow 3)]$ - $\alpha$-L-rhamnopyranosyl- $(1 \longrightarrow 2)-\beta$-D-gluco- pyranoside 20}

Compound 19 (510 mg, $0.27 \mathrm{mmol}$ ) was dissolved in anhydrous $\mathrm{CH}_{2} \mathrm{Cl}_{2}(5 \mathrm{~mL})$ and diluted with absolute methanol $(45 \mathrm{~mL})$. To the above mixture was added sodium methoxide $(0.5 \mathrm{M}$ in $\mathrm{MeOH} ; 0.6 \mathrm{~mL}$ ). The mixture was stirred at room temperature for 4 hours, then neutralized with Dowex $50\left(\mathrm{H}^{+}\right)$, filtered and the filtrate was concentrated under reduced pressure. The residue was purified by Sephadex LH-20 column chromatography $(\mathrm{MeOH})$ to give $\mathbf{2 0}$ as a white solid $(253 \mathrm{mg}, 95 \%)$; $[a]_{\mathrm{D}}^{25}-65\left(c 0.6, \mathrm{CH}_{3} \mathrm{OH}\right) ;{ }^{13} \mathrm{C} \mathrm{NMR}\left(\mathrm{CD}_{3} \mathrm{OD} ; 100 \mathrm{MHz}\right)$ $\delta_{\mathrm{C}} 141.1$ (C-5 of cholesterol), 120.1 (C-6 of cholesterol), 110.3, 105.7, 104.3, 100.8, (C-1 of sugar residues), 85.3, 84.4, 79.7, 79.2, 78.9, 78.4 (2 C), 78.1, 76.8, 75.4, 71.9, 71.2, 70.6, 69.4, $62.9,62.5,62.0$; ESIMS (positive) [Calc. for $\mathrm{C}_{50} \mathrm{H}_{84} \mathrm{NaO}_{19}$ $(M+\mathrm{Na})^{+}:$1011.5. Found: $\left.m / z, 1011.6(\mathrm{M}+\mathrm{Na})^{+}\right]$.

\section{Acknowledgements}

This work was supported by NNSF of China (Projects 29972053, 39970179) and CAS (KIP-RCEES9904, KJ952 J 561 and $\left.\mathrm{KJ} 952 \mathrm{~J}_{1} 510\right)$.

\section{References}

1 The Pharmacopoeia Committee of PRC, Pharmacopoeia (I), People Health Press, Beijing, 1995, pp. 119-120.

2 (a) K. Zou, Y. Zhao, G. Tu, J. Cui, Z. Jia and R. Zhang, Carbohydr. Res., 2000, 324, 182; (b) T. Ikeda, T. Kajimoto, T. Nohara, J. Kinjo and C.-H. Wong, Tetrahedron Lett., 1995, 36, 1509; (c) L. Ma, G. Tu, S. Chen, R. Zhang, L. Lai, X. Xu and Y. Tang, Carbohydr. Res., 1996, 281, 35

3 T. Ikeda, S. Fujiwara, K. Araki, J. Kinjo, T. Nohara and T. Miyoshi, J. Nat. Prod., 1997, 60, 102. 
4 T. Ikeda, T. Kajimoto, J. Kinjo, K. Nakayama and T. Nohara, Tetrahedron Lett., 1998, 39, 3513.

5 (a) Preparative Carbohydrate Chemistry, ed. S. Hanessian, Marcel Dekker, New York, 1996; (b) M. Liu, B. Yu and Y. Hui, Tetrahedron Lett., 1998, 39, 415; (c) M. Liu, B. Yu, Z. Guo, C. Li and Y. Hui, Carbohydr. Lett., 1997, 2, 423; (d) H. Yamada and M. Nishizawa, Tetrahedron Lett., 1987, 28, 4315.

6 (a) S. Deng, B. Yu and Y. Hui, Tetrahedron Lett., 1998, 39, 6511; (b) J. T. Randolph and S. J. Danishefsky, J. Am. Chem. Soc., 1995, 117, 5693.

7 (a) Y. Du and F. Kong, J. Carbohydr. Chem., 1999, 18, 655; (b) Y. Du, Q. Pan and F. Kong, Carbohydr. Res., 2000, 323, 28.

8 (a) E. Petrakova and J. Schraml, Collect. Czech. Chem. Commun., 1983, 48, 877; (b) T. L. Lowary, E. Eichler and D. R. Bundle, J. Org. Chem., 1995, 60, 7316; (c) F.-I. Auzanneau, F. Forooghian and B. M. Pinto, Carbohydr. Res., 1996, 291, 21; (d) W. Wang and F. Kong, Carbohydr. Res., 1999, 315, 128.

9 Y. Du and F. Kong, J. Carbohydr. Chem., 1995, 14, 341

10 S. Saito, S. Sumita, K. Ichinose and Y. Kanda, Chem. Pharm. Bull., 1993, 41, 90.

11 R. R. Schmidt and J. Michel, Angew. Chem., Int. Ed. Engl., 1980, 19, 731

12 R. L. Whistler and M. L. Wolfrom, Methods Carbohydr. Chem., $1963,2,345$.

13 T. W. Greene and P. G. M. Wuts, Protective Groups in Organic Synthesis, Wiley, New York, 1991, pp. 92-93.

14 G. M. Bebault, G. G. S. Dutton and C. K. Warfield, Carbohydr. Res., 1974, 34, 174.

15 Y. Mimaki, M. Kuroda, T. Fukasawa and Y. Sashida, J. Nat. Prod., 1999, 62, 194. 